\title{
THE MAGNETIC FIELD OF THE CRAB AS REVEALED BY NEW, HIGH RESOLUTION, MULTI-BAND RADIO IMAGES
}

\author{
M. F. Bietenholz and P. P. Kronberg \\ Department of Astronomy, University of Toronto, \\ 60 St. George St., Toronto, Ontario, \\ Canada, M5S-1A7.
}

\section{Introduction}

We present and describe recent radio observations of the Crab Nebula, which allow us to determine the magnetic field orientation and depolarization at unprecedented resolution. The observations were made in 1987-1988 using all four configurations of the VLA, at $1410,1515,4625$, and $4885 \mathrm{MHz}$. The resulting maps were all convolved with a clean beam of $1.8^{\prime \prime} \times 2.0^{\prime \prime}$, elongated in P.A. $80^{\circ}$, and the residuals added back in.

\section{The Magnetic Field Configuration}

In order to obtain the magnetic field orientation in the case of Faraday rotation, it is necessary to 'de-rotate' the observed E-vectors to "zero wavelength". Despite the fact that some real deviations from a linear polarization position angle $-\lambda^{2}$ relationship are seen, only small de-rotations are required at $\lambda 6 \mathrm{~cm}$, and the field orientations are relatively secure. The image is shown in Figure 1.

The rotation measure is remarkably uniform over the nebula, $\left(\sim-26 \mathrm{rads} / \mathrm{m}^{2}\right)$ except for small regions of low polarization. This would seem to imply that most of this rotation is due to an external Faraday screen - presumably interstellar rotation between the Crab and us.

Both the jet (variously referred to as the spur, the chimney, or the stovepipe) and the edge of the Nebula are highly polarized, being at least $20-30 \%$ polarized (at $20 \mathrm{~cm}$ ), in contrast to the main body of the nebula which is $\sim 5 \%$ polarized. This indicates that the magnetic field geometry is particularly simple near the edge, where the field also appears to be predominantly radial, possibly suggesting an outward wind.

Several magnetic loops are seen (for example at the south-west edge of the nebula, see Fig. 1) which seem to have no obvious counterpart in I (total intensity). On the other hand, there are loop-like features seen in I, but they don't seem to be related to the field geometry in an obvious way. There are also linear regions of depolarization which are not accompanied by any features in total intensity or rotation measure. These are probably caused by small thermal filaments lying in front of the bulk of the synchrotron emission. At our resolution the I flux seems to be fairly completely resolved (Wilson et al, 1985), whereas the polarization structure does not.

The magnetic field of the jet seems to be directed along its axis (see fig. 1 ). This is the type of field that is expected in the scenarios in which the jet is where relativistic plasma escapes through a hole in the filamentary shell (for example Kundt, 1983, and Schull et al, 1985).

79

R. Beck et al. (eds.), Galactic and Intergalactic Magnetic Fields, 79-80.

() 1990 IAU. Printed in the Netherlands. 


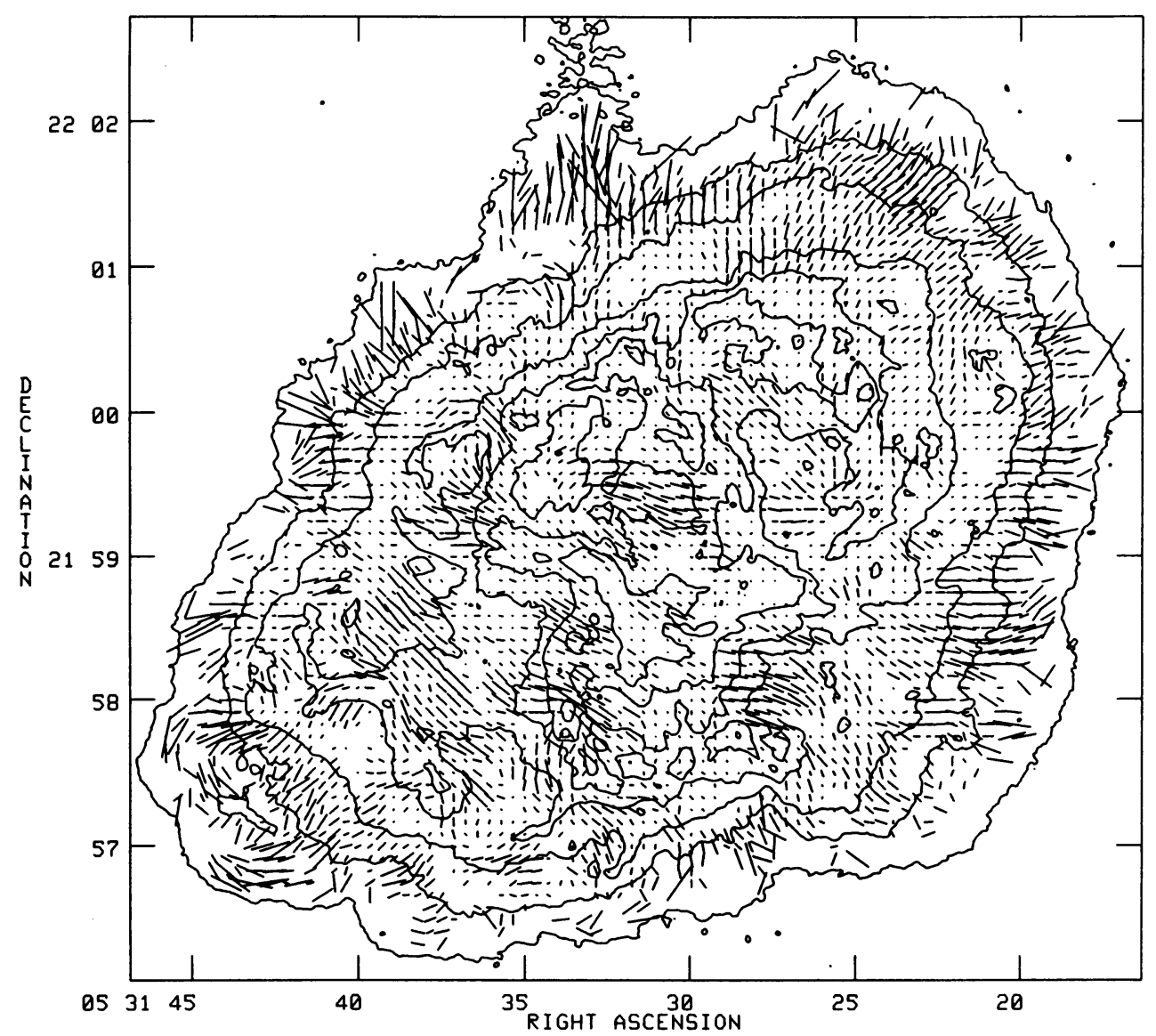

Figure 1: Contour plot representation of the total intensity at $\lambda=20 \mathrm{~cm}$ (contours at $.5,10,20,35,50,65,80 \%$ of the peak flux of $.114 \mathrm{Jy} /$ beam), the direction of the magnetic field and the percentage polarization (a vector length of 50" indicates $100 \%$ polarization). Due to space limitations, polarization vectors are drawn only every 5".

\section{References}

Mclean I. S., et al, 1983. Nature, 304, no. 5923, 243.

Kundt, W., 1983. Astron. and Astroph.,121, L15.

Schull, P., et al, 1984. Ap. J. Lett.,285, L75.

Wilson, A. S., Samarasinha, D. H., Hogg, D. E., 1985. Ap. J. Lett., 294, L121. 